\title{
Desmoplastic small round cell tumor: A case report of a rare differential diagnosis of solid tumors of the pleura
}

\author{
YUAN CAO $^{1 *}$, YING CHEN $^{2 *}$, LI YANG $^{2}$, ZI-HUA QIAN ${ }^{2}$, SHU-GAO HAN ${ }^{2}$, QING-HAI LI ${ }^{2}$ and RI-SHENG YU ${ }^{2}$ \\ Departments of ${ }^{1}$ Pathology and ${ }^{2}$ Radiology, The Second Affiliated Hospital, \\ Zhejiang University School of Medicine, Hangzhou, Zhejiang 310009, P.R. China
}

Received September 19, 2014; Accepted June 16, 2015

DOI: $10.3892 / \mathrm{ol} .2015 .3680$

\begin{abstract}
Desmoplastic small round cell tumor(DSRCT) presents as a rare separate clinical pathological entity, and pleural DSRCT is very rare. Following review of the English literatures it was revealed that, to date, $<15$ cases of primary DSRCT of the pleura have been reported worldwide. Among these, there are few computed tomography (CT) findings of pleural DSRCT which have previously been described in detail. The present study reports a pathologically proven case of pleural DSRCT, with varying contrast CT findings in a 72-year-old female, which appeared as a large $(12.0 \times 10.0 \times 6.5 \mathrm{~cm})$, smooth, oval mass in the left lower thorax with slight-moderate uniform enhancement on contrast-enhanced CT. To the best of our knowledge, the present report is the first to describe the large solid-tumor pattern and the patient is the eldest reported case of pleural DSRCT.
\end{abstract}

\section{Introduction}

Desmoplastic small round cell tumor (DSRCT) is a rare but aggressive primitive malignant neoplasm that occurs mainly in adolescents and young adults (1-3). The abdomen and pelvis are the sites most likely to be involved, while DSRCT of the pleura is even more rare. Review of the English literature revealed that, to date, $<15$ cases of primary DSRCT in the pleura (including the present case) have been reported worldwide (PubMed, http://www.ncbi.nlm.nih.gov/pubmed/) (1-9). Among these cases, there are few computed tomography (CT) findings of pleural DSRCT, which have been previously described in detail $(2,3,8,9)$. The present study describes a rare case of pleural DSRCT with differential contrast CT

Correspondence to: Dr Shu-Gao Han, Department of Radiology, The Second Affiliated Hospital, Zhejiang University School of Medicine, 88 Jiefang Road, Hangzhou, Zhejiang 310009, P.R. China E-mail: hanshugao@163.com

*Contributed equally

Key words: desmoplastic small round cell tumor, pleural neoplasm, solid neoplasm, differential diagnosis, computed tomography findings in a 72-year-old female, and reviews the English literature. Written informed consent was obtained from the patient.

\section{Case report}

A 72-year-old female presented with a history of left-side chest pain, and dyspnea for six months. There were no serious illnesses in the patient's past medical history. Physical examination demonstrated inaudible breath sounds in the left thorax but no other remarkable abnormal findings.

Spiral computed tomography (CT) (Somatom Sensation 16; Siemens, Munich, Germany) findings of the tumor were as follows (Fig. 1A and B): i) Location, the tumor was located in the left lower thorax; ii) shape, the tumor appeared as a large $(12.0 \times 10.0 \times 6.5 \mathrm{~cm})$, smooth, oval mass, which formed obtuse angles with the pleural surface; iii) composition, the tumor appeared homogenous with low attenuation on plain $\mathrm{CT}$ examination, with a mean $\mathrm{CT}$ attenuation value of 28 Hounsfield units (HU); iv) enhancement, the tumor revealed slight-moderate unhomogeneous enhancement on contrast-enhanced CT, with a mean $\mathrm{CT}$ attenuation value of 38HU; and v) neighborhood, the adjacent lung tissues were compressed, and no rib destruction was found, but several enlarged lymph nodes were identified in the mediastinum. Based on these findings, localized fibrous tumor of the pleura was initially considered. Abdominal and pelvic CT scanning identified no neoplasms.

The patient underwent tumor resection. During the operation, the mass was not able to be separated from the pleura, and the basal segment of the left lower lobe was compressed. The tumor size was $\sim 12.0 \times 10.0 \times 6.0 \mathrm{~cm}$, with a smooth surface, and an incomplete capsule.

Microscopically, the tumor was composed of small, round or oval cells, which were generally uniform in size and shape. Most of the cells were closely packed, with transparent cytoplasm, pale nuclei and indistinct nucleoli. No mitotic figures or necrotic cells were detected. The tumor cells were arranged as beam or nest bulk, surrounded by a dense desmoplastic fibrous stroma (Fig. 2); the stroma was rich in vessels, with classic partial thickening of the vascular wall. Immunohistochemical study of the tumor revealed: Pan-cytokeratin (CKpan) (-), CK5/6 (-), epithelial membrane antigen (EMA) (-), carcinoembryonic 

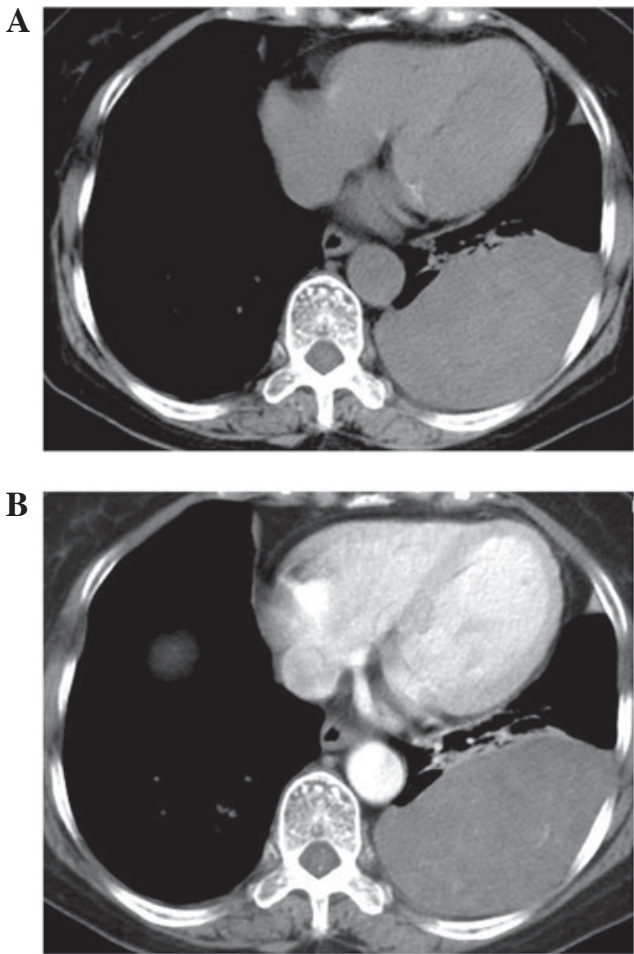

Figure 1. CT examination revealed a large, smooth, solid, oval mass in the left lower thorax, which formed obtuse angles with the pleural surface. (A) The tumor appeared homogenous with low attenuation on plain CT examination, (B) with slight-moderate unhomogeneous enhancement on contrast-enhanced CT. The adjacent lung tissue was compressed. CT, computed tomography.

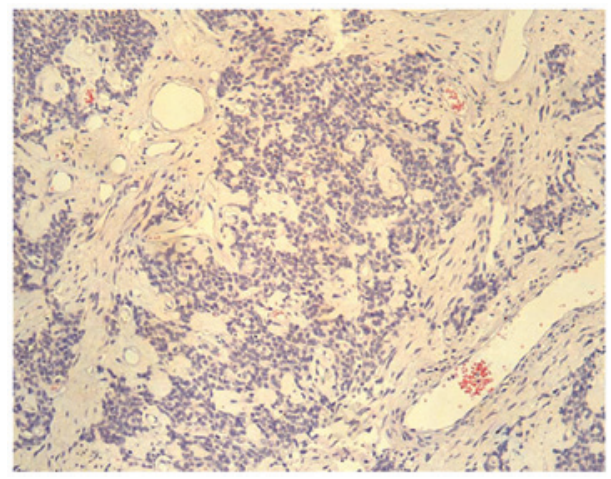

Figure 2. Tumor cells were arranged as beam or nest bulk, surrounded by a dense desmoplastic fibrous stroma, with classic partial thickening of the vascular wall (hematoxylin-eosin stain; magnification, x100).

antigen (CEA) (-), S100 (-), P63 (-), neurofilament protein (NF) (-), CD57 (-), CD99 (+), mesothelial (-), calretinin (+), desmin (-), vimentin (++), smooth muscle actin (SMA) (+), neuron specific enolase (NSE) $(++)$, CD34 (+), CD15 (-), B cell lymphoma-2 (Bcl-2) (+), CD56 (+), chromogranin (CgA) (+), synaptophysin (Syn) (++), adrenocorticotropic hormone (ACTH) (+) and Wilms' tumor (WT-1) (+). Among these factors, vimentin and NSE demonstrated dot-like positive positioning in the nucleus adjacent to the cytoplasm (Fig. 3A-C). Pathological diagnosis of DSRCT of the pleura was ultimately ascertained.

Postoperatively, the patient immediately recovered from her symptoms, refused further chemotherapy or local radiotherapy
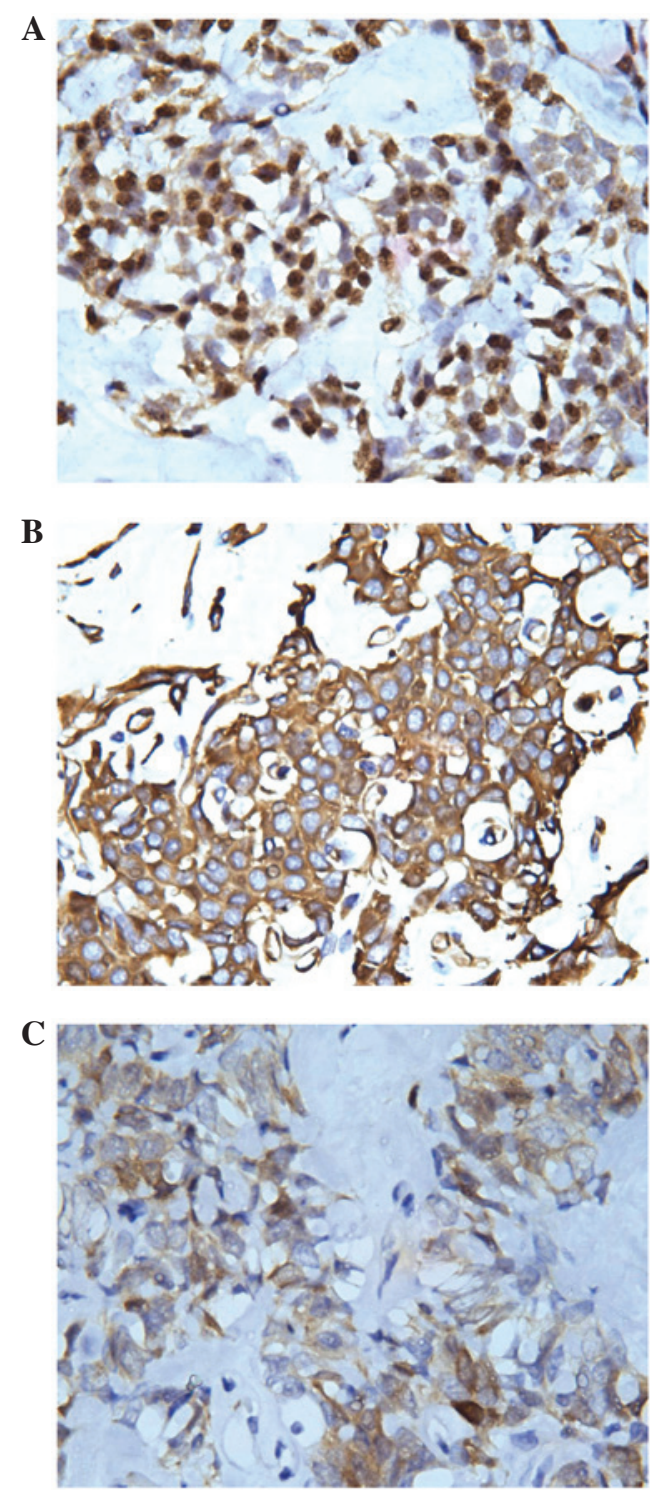

Figure 3. Immunohistochemical staining for (A) WT-1 was positive, while (B) vimentin and (C) NSE revealed dot-like positive positioning in the nucleus adjacent to the cytoplasm (magnification, x250).

and was discharged. There has been no evidence of recurrence or metastasis during the past 32 months of follow-up.

\section{Discussion}

DSRCT is a rare malignancy first described by Gerald and Rosai (1) in 1989. DSRCT is characterized by aggressive behavior and poor prognosis, with a predominance amongst male patients and increased frequency during the second and third decades of life (5). Typically, the abdomen and pelvis are the sites most likely to be involved in DSRCT. DSRCT of the pleura is extremely rare and, to the best of our knowledge, $<15$ cases of primary DSRCT in the pleura (including the present case) have been reported worldwide (PubMed, http://www.ncbi.nlm.nih.gov/pubmed/) (1-9). The majority of these cases have been reported in adolescents or young adults, while the eldest patient in the published literatures was a 29 -year-old male. The present article described the case of 72-year-old female with DRSCT in the left pleural 


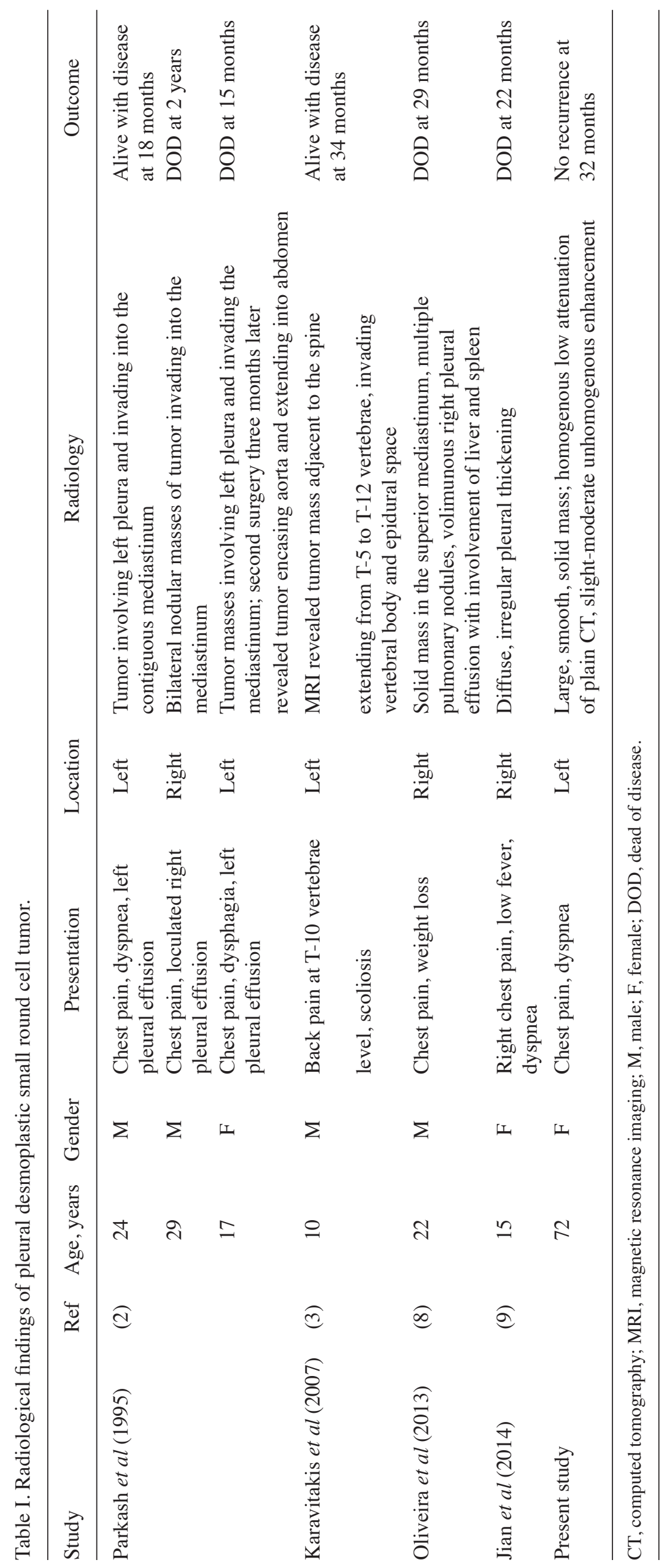


cavity, which therefore represents the eldest patient in the literature to date. The most common presentation of patients with DSRCT of the pleura is that of nonspecific chest pain and respiratory symptoms $(2,3)$. The primary symptom in the present case was comparable with that of previous reports. Certain patients may have a history of exposure to asbestos, smoking and exposure to jute (2). No risk factors or specific causes were identified in the present case.

Histologically, DRSCTs are characterized by nests of small round tumor cells embedded in a dense desmoplastic fibrous stroma. Immunohistochemically, the primary diagnostic feature of DRSCTs is the coexpression of epithelial, mesenchymal and neural cell markers, supported by molecular studies which have identified a specific translocation $\mathrm{t}(11 ; 22)$ (p13; q12) unique to this neoplasm $(4,5)$. Gerald et al (5) indicated that genetic studies are essential for accurate diagnosis in unclear cases, since these may identify the characteristic Ewing's sarcoma (EWS)/WT-1 gene fusion product, which induces transcriptional activation and facilitates uncontrollable growth of tumor cells (10). The immunohistochemical results of the present study were mainly consistent with previous observations. However, the present case of DRSCT was negative for epithelial cell markers and fewer mitotic figures were identified, compared with that of previous reports $(2,3,8,9)$. It was hypothesized that these two factors may be associated with the relatively good prognosis of the patient in the present case. Unfortunately genetic analysis was not performed in the present case, so it remains unknown as to whether the patient possessed the EWS/WT-1 gene fusion.

A number of radiological findings in pleural DSRCT have previouslybeendescribed $(2,3,8,9)$; however,contrast-enhanced CT findings have not been well addressed. According to the literature on pleural DSRCT, radiological manifestations are variable $(2,3,8,9)$. Jian et al (9) and Parkash et al (2) reported diffuse irregular or nodular pleural thickening, as well as pleural effusion of pleural DSRCT on plain CT in adolescents and young adults. Oliveira et al (8) identified a solid mass in the superior mediastinum, multiple pulmonary nodules and a voluminous right pleural effusion, with involvement of the liver and spleen. Furthermore, Karavitakis et al (3) reported a pediatric case of primary pleural DSRCT, presenting with a solid paraspinal lesion extending from vertebrae T-5 to T-12 and invading the 9 th and 10th vertebral bodies, posterior section of the left analogue ribs and epidural space, which was identified by magnetic resonance imaging. In the present case, the tumor demonstrated differential imaging findings. The tumor presented as a large, smooth, oval-shaped solid mass in the left lower thorax, with slight-moderate unhomogenous enhancement on contrast-enhanced CT. It was suggested that the slight-moderate enhancement on the contrast CT scan may be associated with the dense desmoplastic fibrous stroma around the tumor cells. To the best of our knowledge, these large solid-mass radiological manifestations have not previously been described in English literatures. Unfortunately, no two-phase contrast scan was used in the present study, so whether the tumor may be further reinforced in a two-phase contrast scan remains unknown, and further studies are required in the future. All these imaging features are summarized in Table I.
There are numerous differential diagnoses that must be considered upon detection of a pleural mass. Pleural DSRCT mainly requires differentiation from pleural malignant mesothelioma and localized fibrous tumor of the pleura. Previous studies (11-13) indicated that the typical CT results of pleural malignant mesothelioma include unilateral pleural effusion and thickening of the mediastinal pleura, as well as circumferential and nodular pleural thickening of $>1 \mathrm{~cm}$ with mild enhancement, in addition to interlobar fissure thickening. Localized fibrous tumor of the pleura typically presents as a smooth, round or oval, homogeneous mass, with intermediate to high attenuation on unenhanced CT scans. In cases of particularly large lesions, contrast enhancement may be heterogeneous with central areas of low attenuation that correspond with myxoid alterations, hemorrhage, necrosis or cystic degeneration (13-15).

Although the patient in the present study refused further chemotherapy or local radiotherapy and had no evidence of recurrence or metastasis during 32 months of follow-up, long-term survivors have been reported in the literature mainly as a result of multidisciplinary treatments, including chemotherapy, surgery and radiotherapy $(7,16)$.

Although pleural DSRCT is rare and the final diagnosis depends on histopathology or gene analysis, it should be considered in the differential diagnosis list of large solid masses of the pleura with slight-moderate enhancement on contrast CT, particularly when there is insufficient evidence for the diagnosis of malignant pleural mesothelioma, localized fibrous tumor of the pleura or other relatively common diseases of the pleura in adolescents and young adults.

\section{References}

1. Gerald WL and Rosai J: Case 2. Desmoplastic small cell tumor with divergent differentiation. Pediatr Pathol 9: 177-183, 1989.

2. Parkash V, Gerald WL, Parma A, Miettinen M and Rosai J: Desmoplastic small round cell tumor of the pleura. Am J Surg Pathol 19: 659-665, 1995.

3. Karavitakis EM, Moschovi M, Stefanaki K, Karamolegou K, Dimitriadis E, Pandis N, Karakousis CP and Tzortzatou-Stathopoulou F: Desmoplastic small round cell tumor of the pleura. Pediatr Blood Cancer 49: 335-338, 2007.

4. Ordóñez NG: Desmoplastic small round cell tumor: I: A histopathologic study of 39 cases with emphasis on unusual histological patterns. Am J Surg Pathol 22: 1303-1313, 1998.

5. Gerald WL, Ladanyi M, de Alava E, Cuatrecasas M, Kushner BH, LaQuaglia MP and Rosai J: Clinical, pathologic and molecular spectrum of tumors associated with $t(11 ; 22)$ (p13;q12): Desmoplastic small round-cell tumor and its variants. J Clin Oncol 16: 3028-3036, 1998.

6. Hayes-Jordan A and Anderson PM: The diagnosis and management of desmoplastic small round cell tumor: A review. Curr Opin Oncol 23: 385-389, 2011.

7. Kushner BH, LaQuaglia MP, Wollner N, Meyers PA, Lindsley KL, Ghavimi F, Merchant TE, Boulad F, Cheung NK, Bonilla MA, et al: Desmoplastic small round-cell tumor: Prolonged progression-free survival with aggressive multimodality therapy. J Clin Oncol 14: 1526-1531, 1996.

8. Oliveira MJ, de Almeida LP, Wengerkievicz AC, Siqueira SA and Antonangelo L: From conventional fluid cytology to unusual histological diagnosis: Report of four cases. Diagn Cytopathol 41: 348-353, 2013.

9. Jian Z, Shaohong H, Wenzhao Z and Lijia G: Misdiagnosed desmoplastic small round cell tumor of the pleura: Case report and literature review. J Formos Med Assoc 113: 60-61, 2014.

10. Sandberg AA and Bridge JA: Updates on the cytogenetics and molecular genetics of bone and soft tissue tumors, desmoplastic small round-cell tumors. Cancer Genet Cytogenet 138: 1-10, 2002. 
11. Kawashima A and Libshitz HI: Malignant pleural mesothelioma: CT manifestations in 50 cases. AJR Am J Roentgenol 155: 965-969, 1990

12. Wang ZJ, Reddy GP, Gotway MB, Higgins CB, Jablons DM Ramaswamy M, Hawkins RA and Webb WR: Malignant pleural mesothelioma: Evaluation with CT, MR imaging and PET. Radio Graphics 24: 105-119, 2004.

13. Jeong YJ, Kim S, Kwak SW, Lee NK, Lee JW, Kim KI, Choi KU and Jeon TY: Neoplastic and nonneoplastic conditions of serosal membrane origin: CT findings. Radiographics 28 : 801-817, 2008.
14. Mendelson DS, Meary E, Buy JN, Pigeau I and Kirschner PA: Localized fibrous pleural mesothelioma: CT findings. Clin Imaging 15: 105-108, 1991

15. Dedrick CG, McLoud TC, Shepard JA and Shipley RT: Computed tomography of localized pleural mesothelioma. AJR Am J Roentgenol 144: 275-280, 1985.

16. Kurre P, Felgenhauer JL, Miser JS, Patterson K and Hawkins DS: Successful dose-intensive treatment of desmoplastic small round cell tumor in three children. J Pediatr Hematol Oncol 22: 446-450, 2000 